\title{
Maker-Plastic In, Plastic Out: Circular Economy and Local Production
}

\author{
Asger Nørregård-Rasmussen, Malte Hertz-Jansen, \\ and Felicitas Schmittinger
}

Recognising the lack of local and economically accessible facilities, technologies, and public engagement in local recycling, the chapter tackles the challenge of introducing Circular Economy to cope with plastic waste in Copenhagen. The need for circular systemic innovation and holistic production models for recycling plastics led to consider how local micro entrepreneurs, SMEs, commercial resellers and citizens can collaborate for a common, sustainable goal. The chapter presents 'Plastic In, Plastic Out' (PIPO), a Circular system for local sourcing, recycling and production of sustainable plastic building materials and products.

\section{Introduction}

\section{The organisation}

Maker is a FabLab located in Copenhagen (Denmark) with a focus on local production, circular economy and the democratisation of knowledge.

Founded in 2015 it addressed challenges of local and national relevance by fostering the maker-ecosystem and creating and nourishing a network among makers, enterprises, public entities, civil society and policymakers.

This is established by applying the co-creation approach to the development of new, sustainable solutions applying design thinking tools and building new capacities

\footnotetext{
A. Nørregård-Rasmussen $(\varangle) \cdot M$. Hertz-Jansen

Maker/Viadukten, Copenhagen, Denmark

e-mail: asger@maker-effekt.dk

M. Hertz-Jansen

e-mail: malte@maker-effekt.dk

F. Schmittinger

Department of Design, Politecnico di Milano, Milan, Italy

e-mail: felicitas.schmittinger@polimi.it
} 
in relation to the co-creation of solutions, prototyping and the application of new technologies.

Maker supports makers and entrepreneurs as well as local actors and stakeholders in Denmark as a mediator fostering formal and informal connections and relationships contributing to strengthen local landscapes of stakeholders in Denmark.

\section{The co-creation journey}

The provisioning, consumption and disposal of rising amounts of resources is immensely challenging countries and regions worldwide. Denmark, and Copenhagen in particular, counts a waste production per capita double the European average [1] leading to the development of the Resource and Waste Management Plan 24 (RAP 24) that clearly states the pressing need for change in the following years $[2,3]$.

At the same time, alternative models for the local production and recycling of resources are emerging and citizen-driven initiatives have started tackling the issue.

Maker addresses this need for more sustainable solutions with a focus on circular economy instead of traditional linear production chains. Aiming to enhance local cooperation and collaboration and the involvement of fablab and maker communities, the prototype takes a focus on the creation of a new approach towards a communitydriven ecosystem enabling new models and systems for the recycling of plastic waste.

It was developed over the course of 21 months by analysing the current local systems and infrastructures to create a shared knowledge base and elaborate a new, integrated ecosystem of stakeholders in Copenhagen.

'Plastic In, Plastic Out' (PIPO) integrates with the existing ecosystem offering an alternative value-chain by producing plastic sheets and products made of recycled plastic combining the reuse, recycling, manufacturing and consumption by adopting a local and systemic approach.

\section{Ecosystem, Context and Challenge Addressed}

\section{Ecosystem}

Denmark's recycling rate is lower than the average in Europe, especially the direct recycling and re-use of materials is complicated by a variety of legislative obstacles omitting the development of local recycling systems.

It has been acknowledged that not only legislative change is needed, but also the socio-cultural aspect and the specificities of the local contexts and environments need to be taken into account when moving towards the more efficient use of resources.

These particular contexts need to be explored and understood in depth to then develop new technical and innovative solutions to foster the ongoing trend of recycling the highest amount of waste possible through the improvement of waste sorting and management that has risen from 27 to over $45 \%$ in the last years in the city of Copenhagen showing a positive trend [2]. 
The concrete goals of the city to improve the use of resources and waste throughout the next year aligns Maker's goals with the local agendas producing a series of other activities and projects with similar scopes to jointly obtain an adaptation of limiting regulations related to regulations for material reuse, certifications and material transparency.

The political landscape is currently undergoing an important process of transformation acknowledging not only the importance to adapt policies, but also to involve citizens and stakeholders to provoke behavioural change and collaboration among the actors towards a common goal.

\section{Organisational context}

Maker as a FabLab has varied experiences in facilitating and fostering cross-sector collaboration to promote entrepreneurship, open source management and initiatives related to circular economy.

As a part of the maker movement and the FabLab network since 2015, the 'maker mindset' consists of elements from design thinking, prototyping and iterative design methods applied regularly both for the exploration of topics and the validation of concepts.

A wide variety of stakeholders are involved in their projects ranging from architects and designers to civil servants and policymakers. The aforementioned 'maker mindset' and their set of collaborative techniques and tools allow the collaboration in a wide network of different stakeholders exploiting and integrating varied knowledge and experiences to develop solutions collaboratively.

Co-creation and co-design as well as a variety of prototyping techniques are applied in formal or informal, iterative processes that are adapted to the specific project and stakeholders involved to create common ground.

\section{Challenge}

Starting from the Fab City agenda, Maker aimed to develop a small-scale circular ecosystem with a high potential for replication and scaling establishing a community for local production and circular economy [4]. Having identified the increasing requests for locally produced plastic together with a rising number of designers and makers buying recycled plastic sheets from the UK-based company SMILE plastics.

Maker's challenge consists in developing new possibilities for local recycling of plastic waste in Copenhagen by addressing the lack of adequate facilities, knowhow and entire systems of recycling and production of new materials.

An entirely new chain of production needs to be developed identifying generators of plastic waste to opportunities for recycling and the creation of new materials for the use and processing of these materials taking an entirely new chain and ecosystem of recycling and production into account. 


\section{The Co-creation Journey}

\subsection{Context Analysis}

Starting from a desk research on the various aspects of the chosen issue like circular economy, plastic production, recycling of plastic, community-driven solutions, development plans and strategies and the national and regional legislations in relation to them, already a number of stakeholders from industry and the innovation community have been involved.

The identified stakeholders and stakeholder groups have then been mapped within an ecosystem model dividing them into categories (Fig. 1) and to be updated after every step of the co-creation process.

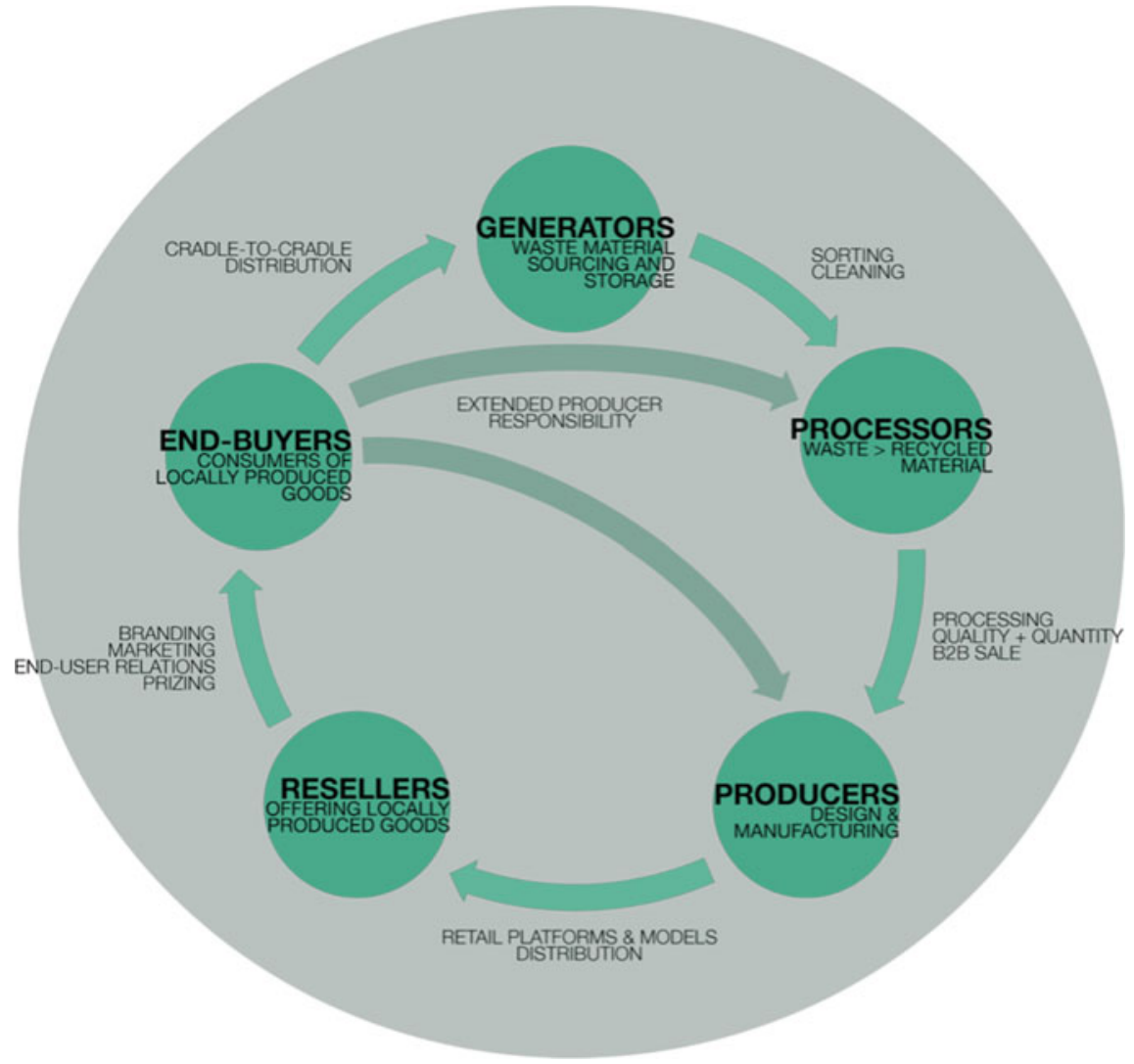

Fig. 1 Initial draft of a local cradle-to-cradle ecosystem model (Elaborated by Stine Broen Christensen) 
Further field research conducted through interviews, workshops and field visits led to further definition of the context and field of research to frame the precise challenge and build a common base to start developing a common solution.

\section{Problem reframing}

The problem has been precisely defined according to three main points:

1. Theoretical aspects and agenda from the Fab City initiative-i.e. creating more livable cities and community focused descriptions [4].

2. Direct feedback on the effective scaling opportunities, industry collaborations and technical solutions.

3. A shared intent to focus more on empowerment, community building, open access to knowledge and learnings.

In collaboration with a group of students, the entire lifecycle of plastic has been investigated and assessed to then interact with industrial stakeholders and public employees to grasp the challenge from a variety of different perspectives conducting a series of workshops, interviews and informal meetings (Fig. 2).

\section{Envisioning of alternatives}

Starting from a series of prototyping activities for the recycling and reuse of plastic the phase of solution development has been initiated.

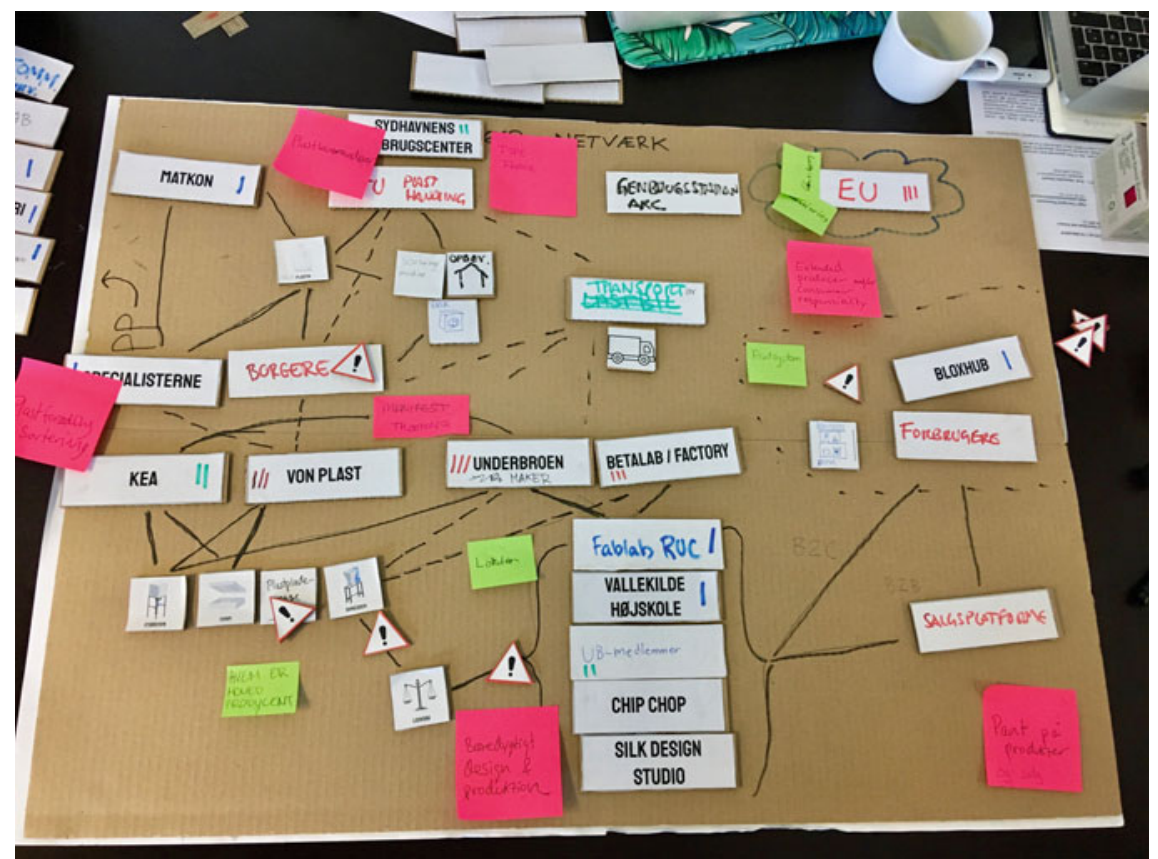

Fig. 2 Game and stakeholder mapping by AAU students \& Maker 
A set of co-design workshops, a maker meet-up and an open lab day have been organised to refine the concept and make concrete plans for the prototyping phase as well as aligning the solution to the needs of all stakeholders.

In this phase, also the FabCity initiative has been involved again through talks at the Open Lab Day to reconnect the prototype to their general agenda and inspire local stakeholders.

In this process, the concept has been narrowed down with a focus on the recycling of plastic for the co-production of recycled plastic sheets and their processing into products integrating this new ecosystem in the current network of circular economy in Copenhagen aligning it with existing initiatives, municipal projects and creating collaborative relations with the involved stakeholders (Fig. 3).

\section{Development and prototyping}

The prototyping process underwent several iterations to improve both the plastic sheets as the produced material and core of the prototype as well as the ecosystem developed to meet all stakeholders' needs.

9 different products have been produced locally from the plastic sheets that then have been showcased in an exhibition taking place digitally due to the COVID-19 pandemic.

Apart from the incubation of the production of new objects made from recycled plastic, Maker closely collaborated with the industry and innovation community as well as policymakers organising educational activities and co-design workshops engaging more than 150 participants. This has supported the integration with

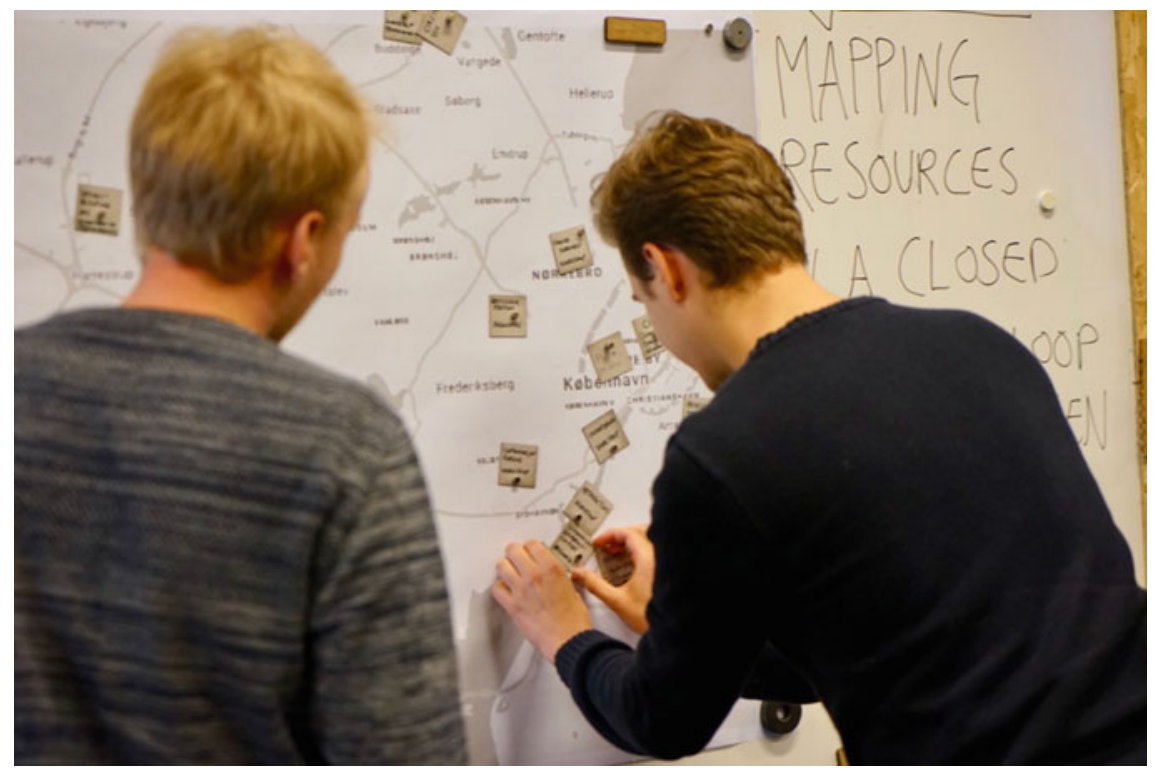

Fig. 3 Maker Meet Up and Open Lab Day in May 2019 
a

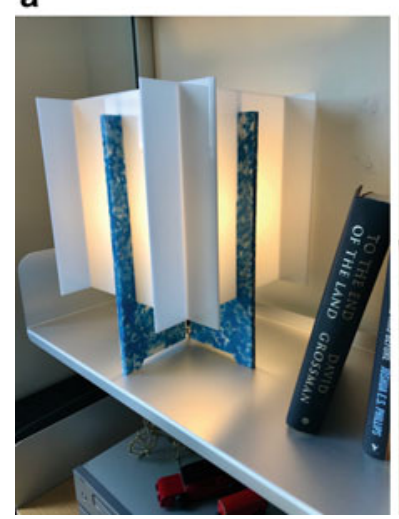

b

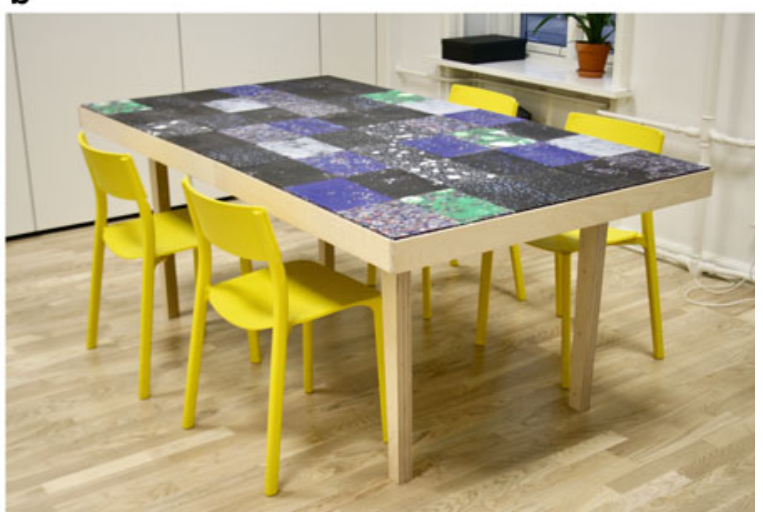

Fig. 4 a, b Projects developed as part of the prototype

local agendas and initiatives while raising awareness on the need for policy change, building a community and identifying challenges and opportunities for scaling and replication (Fig. 4).

\section{Experimentation: Output, Transformations, Outcomes}

PIPO stands for 'Plastic In, Plastic Out' and describes a new ecosystem model for small-scale circular economy for the recycling and reuse of plastic with local actors. It aims to connect local initiatives to municipal agendas while showcasing concrete results in a gallery of prototypes to inspire and further develop the use of the material. It functions as an example as a part of the FabCity initiative as well as a way of introducing circular economy in a specific context in an ecosystem empowering local makers and designers.

\section{Transformations}

Maker as an organisation has faced a large impact on a strategic level leading to a reorientation towards circular economy and community building through co-creation caused by the project. The previous experience with cross-sector collaborations and co-creation has been enriched and strengthened by the provided framework and training leading to an internal professionalisation and establishment of practices.

Furthermore, the organisation has established new networks and contacts fostering a multi-stakeholder environment and creating a perspective for future collaborations and projects.

The project led to a raised awareness of plastic waste and opportunities to tackle the issue within the core group of stakeholders triggering a series of similar initiatives and considerations for replication. 
The COVID-19 pandemic has significantly challenged the execution and activities enabling flexibility, alternative solutions and capacity to cope with challenges and complexity.

\section{Scaling}

Being based on the overall challenge to fight plastic waste on a large scale, considerations have been made on scaling the locally developed systems.

The main aim is to exploit the learnings and knowledge gained for the development of other circular models, reuse the process developed in other projects and provide this knowledge open access to empower other communities to establish similar ecosystems.

The scaling of the material as the technical part of the solution has been assigned less importance in this case following an analysis of potential and possibilities with stakeholders and policymakers identifying the difficulty of scaling 'maker solutions' to an industrial level and recognising the development of the ecosystem as the more promising part of the developed solution.

\section{Lessons Learnt and Reflections}

Co-creation has been identified as a trigger not only to guide activities, but to generate interest by fostering communication, cooperation and inspiration. For the co-creation activities conducted, the importance and benefits of the variety of tools provided has resulted as fundamental in supporting the planning, leading and guidance of workshops and sessions.

Tensions and difficulties of communication among stakeholders and stakeholder groups have been traced back to their inequality in terms of levels and positions raising the need to implement further mechanisms to balance this contrast.

The difficulties in engaging policymakers as a stakeholder group resulted as a barrier, since their engagement at the same time has been found crucial to shape a realistic and implementable solution.

\section{References}

1. Danmark Uden Affald: genanvend mere - forbrænd mindre. Miljøstyrelsen MST 2011, https:// mst.dk/media/mst/Attachments/MST_Faktaark_1_WEB.pdf. Last accessed 2021/01/05

2. Cirkulær København: Resource- og Affaldsplan 2024. Københavns Kommune 2019, https://kk. sites.itera.dk/apps/kk_pub2/index.asp?mode $=$ detalje\&id=1990. Last accessed 2021/01/05

3. KBH 2025 Klimaplanen - Københavns Kommune 2012, https://kk.sites.itera.dk/apps/kk_pub2/ index.asp?mode=detalje \&id=1035. Last accessed 2021/01/05

4. Diez et al. Fab City Whitepaper: Locally productive, globally connected self-sufficient cities, https://fab.city/uploads/whitepaper.pdf. Last accessed 2021/01/05 
Open Access This chapter is licensed under the terms of the Creative Commons Attribution 4.0 International License (http://creativecommons.org/licenses/by/4.0/), which permits use, sharing, adaptation, distribution and reproduction in any medium or format, as long as you give appropriate credit to the original author(s) and the source, provide a link to the Creative Commons license and indicate if changes were made.

The images or other third party material in this chapter are included in the chapter's Creative Commons license, unless indicated otherwise in a credit line to the material. If material is not included in the chapter's Creative Commons license and your intended use is not permitted by statutory regulation or exceeds the permitted use, you will need to obtain permission directly from the copyright holder.

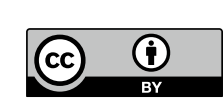

